M. Takeuchi

Nagoya Math. J.

Vol. 115 (1989), 43-46

\title{
TWO-NUMBER OF SYMMETRIC R-SPACES
}

\author{
MASARU TAKEUCHI
}

\section{Dedicated to Professor Shingo Murakami on his sixtieth birthday}

\section{Introduction}

Chen-Nagano [2] introduced a Riemannian geometric invariant $\nu(M)$, called the 2-number, for a compact (connected) symmetric space $M$ : Points $p, q \in M$ are said to be antipodal to each other, if $p=q$ or there is a closed geodesic of $M$ on which $p$ and $q$ are antipodal to each other. A subset $A$ of $M$ is called an antipodal subset if every pair of points of $A$ are antipodal to each other. Now the 2-number $\nu(M)$ is defined as the maximum possible cardinality $|A|$ of an antipodal subset $A$ of $M$. The 2-number is finite.

In this note we will prove the following

TheOREm. If $M$ is a symmetric $R$-space (See $\S 1$ for the definition), we have

$$
\nu(M)=\operatorname{dim} H\left(M, Z_{2}\right),
$$

where $H\left(M, Z_{2}\right)$ denotes the homology group of $M$ with coefficients $Z_{2}$.

\section{§1. Symmetric $R$-spaces}

A compact symmetric space $M$ is said to have a cubic lattice if a maximal torus of $M$ is isometric to the quotient of $\boldsymbol{R}^{r}$ by a lattice of $\boldsymbol{R}^{r}$ generated by an orthogonal basis of the same length. A Riemannian product of several compact symmetric spaces with cubic lattices is called a symmetric $R$-space. We here recall some properties of symmetric $R$ spaces (cf. Takeuchi [4], [6], Loos [2]).

A symmetric $R$-space $M$ has the complexification $\bar{M}$ : There exists uniquely a connected complex projective algebraic manifold $\bar{M}$ defined over $\boldsymbol{R}$ such that the set $\bar{M}(\boldsymbol{R})$ of $\boldsymbol{R}$-rational points of $\bar{M}$ is identified

Received July 22, 1988. 
with $M$. The group Aut $\bar{M}$ of holomorphic automorphisms of $\bar{M}$ is a complex linear algebraic group defined over $\boldsymbol{R}$. The identity component $G$ of $($ Aut $\bar{M})(R)$ is a semi-simple Lie group without compact factors and acts on $M$ effectively and transitively. The identity component $K$ of the group $\mathrm{I}(M)$ of isometries of $M$ is a maximal compact subgroup of $G$. Thus there is an involutive automorphism $\tau$ of $G$ such that

$$
K=\{g \in G ; \tau(g)=g\} .
$$

We fix a point $o \in M$ once and for all, and set

$$
U=\{g \in G ; g \cdot o=o\}, \quad K_{0}=K \cap U .
$$

Thus we have identifications: $M=G / U=K / K_{0}$. Let

$$
\mathfrak{g}=\mathfrak{l}+\mathfrak{p}, \quad \mathfrak{t}=\operatorname{Lie} K
$$

be the eigenspace decomposition of $g=\operatorname{Lie} G$ with respect to the differential of $\tau$. Then there exists uniquely an element $E \in \mathfrak{p}$ such that $\mathfrak{u}=$ Lie $U$ is given by

$$
\mathfrak{u}=\mathfrak{g}_{0}+\mathfrak{g}_{1},
$$

where $\mathfrak{g}_{p}$ denotes the $p$-eigenspace of ad $E$. Furthermore the subgroup $K_{0}$ coincides with the centralizer of $E$ in $K$, and so $M$ is imbedded into $\mathfrak{p}$ as the $K$-orbit through $E$. We choose a maximal abelian subalgebra $a$ in $\mathfrak{p}$ with $E \in \mathfrak{a}$ and set

$$
W=N_{K}(\mathfrak{a}) / Z_{K}(\mathfrak{a}), \quad W_{0}=N_{K_{0}}(\mathfrak{a}) / Z_{K_{0}}(\mathfrak{a}),
$$

where $N_{K}(\mathfrak{a})$ (resp. $\left.N_{K_{0}}(\mathfrak{a})\right)$ and $Z_{K}(\mathfrak{a})$ (resp. $Z_{K_{0}}(\mathfrak{a})$ ) denote the normalizer and the centralizer in $K$ (resp. in $K_{0}$ ) of $a$. We may regard $W_{0}$ as a subgroup of $W$. These groups $W$ and $W_{0}$ are finite groups called Weyl groups of $\mathfrak{g}$ and $g_{0}$. We define a subset $A$ of $M$ by

$$
A=N_{K}(\mathfrak{a}) \cdot o \text {. }
$$

Since $A \cong N_{K}(\mathfrak{a}) / N_{K_{0}}(\mathfrak{a}) \cong W / W_{0}$, we have

$$
|A|=\left|W / W_{0}\right| \text {. }
$$

Theorem 1. (Bott-Samelson [1]).

$$
\operatorname{dim} H\left(M, Z_{2}\right)=\left|W / W_{0}\right| \text {. }
$$

Theorem 2 (Takeuchi [4]). Let $M_{1}, \cdots, M_{s}$ be the connected compo- 
nents of fixed point set of the symmetry of $M$ at o. Thus each $M_{i}$ is a compact symmetric space with respect to the Riemannian metric induced from that of $M$. Then

(i) Each $M_{i}$ is also a symmetric $R$-space; and

(ii) $\operatorname{dim} H\left(M, Z_{2}\right)=\sum_{i=1}^{s} \operatorname{dim} H\left(M_{i}, Z_{2}\right)$.

TheOREM 3 (Takeuchi [4], [5]). There exists a maximal torus of $M$ through o which includes antipodal points $a_{i}(1 \leq i \leq s)$ to o such that

$$
A=N_{K_{0}}(\mathfrak{a}) \cdot\left\{a_{1}, \cdots, a_{s}\right\}
$$

\section{§2. Proof of Theorem}

We first show that the subset $A$ is an antipodal subset. We remark that since $K_{0} \subset \mathrm{I}(M)$ and $N_{K_{0}}(\mathfrak{a}) \cdot o=\{o\}$, by Theorem 3 each point of $A$ is antipodal to $o$. Let $p, q \in A$ be arbitrary. Since $A=N_{K}(\mathfrak{a}) \cdot o$, there is $k \in N_{K}(\mathfrak{a})$ such that $k \cdot p=0$. By the above remark the point $k \cdot q \in A$ is antipodal to $o=k \cdot p$. It follows by $k \in \mathrm{I}(M)$ that $q$ is antipodal to $p$. This proves the claim. Now, together with Theorem 1 this implies the inequality:

$$
\operatorname{dim} H\left(M, Z_{2}\right) \leq \nu(M)
$$

We will prove Theorem by induction on $\operatorname{dim} M$. We make use of the inequality in Chen-Nagano [2]:

$$
\nu(M) \leq \sum_{i=1}^{s} \nu\left(M_{i}\right)
$$

which holds for a general compact symmetric space. By Theorem 2 (i) and the assumption of the induction we have that $\nu\left(M_{i}\right)=\operatorname{dim} H\left(M_{i}, Z_{2}\right)$ for each $i$, and hence

$$
\nu(M) \leq \sum_{i=1}^{s} \operatorname{dim} H\left(M_{i}, Z_{2}\right) .
$$

But the right hand side is equal to $\operatorname{dim} H\left(M, Z_{2}\right)$ by Theorem 2 (ii), and so we obtain the inequality:

$$
\nu(M) \leq \operatorname{dim} H\left(M, Z_{2}\right) .
$$

Together with the previous opposite inequality we get

$$
\nu(M)=\operatorname{dim} H\left(M, Z_{2}\right) .
$$




\section{REFERENCES}

[1] R. Bott and H. Samelson, Applications of the theory of Morse to symmetric spaces, Amer. J. Math., 80 (1958), 964-1029.

[2] B.-Y. Chen and T. Nagano, A Riemannian geometric invariant and its applications to a problem of Borel and Serre, Trans. Amer. Math. Soc., 308 (1988), 273-297.

[ 3 ] O. Loos, Charakterisierung symmetrischer $R$-Räume durch ihre Einheitsgitter, Math. Z., 189 (1985), 211-226.

[4] M. Takeuchi, Cell decompositions and Morse equalities on certain symmetric spaces, J. Fac. Sci. Univ. Tokyo, 12 (1965), 81-192.

[5] — On orbits in a compact hermitian symmetric space, Amer. J. Math., 90 (1968), 657-680.

[ 6 ] — Basic transformations of symmetric $R$-spaces, Osaka J. Math., 25 (1988), 259-297.

Department of Mathematics

College of General Education

Osaka University

Toyonaka 560, Japan 